Rev. Elet. em Gestão, Educação e Tecnologia Ambiental (e-ISSN: 2236-1170)

\title{
VIABILIDADE ECONÔMICA DE UM SISTEMA DE TERMINAÇÃO DE CORDEIROS EM CONFINAMENTO NA REGIÃO DA CAMPANHA/RS
}

\author{
ECONOMICAL VIABILITY OF A LAMBS FINISHING SYSTEM IN THE CAMPAIGN REGION / RS \\ Milene Piccoli, Gladis Ferreira Corrêa, Júlio Eduardo Rohenkohl, Jalise Fabíola Tontini, Sheilla \\ Madruga Moreira, Marivane Vestena Rossato
}

http://dx.doi.org/10.5902/223611708818

RESUMO

O trabalho foi desenvolvido entre os meses de março e junho, na propriedade particular de um membro da Associação de Criadores de Ovinos de Dom Pedrito (ACODOPE). Foram avaliados 60 cordeiros, 50 animais da raça Corriedale e 10 da raça Texel, todos machos castrados, dente de leite, com cinco a sete meses de idade. Buscou-se no decorrer do experimento avaliar o custo de implantação de um sistema de confinamento e sua lucratividade. Os animais foram pesados ao longo do experimento para o controle do ganho de peso. Para atingir os resultados exatos da produção, foram avaliados e representados todos os custos de implantação e funcionamento do confinamento como: Custos Fixos, Custos Variáveis e o Custo de aquisição dos animais. Foi observado que o os animais do lote Texel permaneceram menos tempo para atingir o peso de abate, conseqüentemente o custo de produção dos mesmos foi inferior aos demais. Mesmo assim apresentaram um prejuízo de $\mathrm{R} \$ \mathbf{0 , 6 9}$ por $\mathrm{Kg}$ de peso vivo. Resultados negativos foram encontrados nos outro dois lotes, sendo que o lote com aplicação de Fósforo Orgânico associado a Vitamina $B_{12}$ $\left(F B_{12}\right)$ apresentou um prejuízo de $\mathrm{R} \$ 1,77$ por $\mathrm{Kg}$ de peso vivo, e o lote dos animais sem aplicação de $\mathrm{FOB} \mathrm{B}_{12}, \mathrm{R} \$ \mathbf{0 , 7 5}$ por $\mathrm{Kg}$ de peso vivo. Tais resultados tornaram o sistema de terminação de cordeiros em confinamento, testado nesta experimentação, inviável economicamente.

Palavras-chave: custos, lucratividade, terminação de cordeiros

\begin{abstract}
The study was conducted between March and June in a particular property of a member of the Association of Sheep Breeders of Dom Pedrito (ACODOPE). Sample consisted of 60 lambs, 50 animals and 10 Corriedale breed Texel, all barrows, milk tooth, with five to seven months old. Was sought throughout the experiment to assess the cost and profitability of a lambs finishing system. The animals were weighed throughout the experiment for the control of weight gain. To achieve the exact results of the production were evaluated and represented all the costs of implementation and operation of confinement as: Fixed Costs, Variable Costs and Cost of acquisition of animals. It was observed that the animals Texel lot remained less time to reach slaughter weight, therefore the cost of producing them was inferior to the other. Still had a loss of R\$ 0.69 per kg bodyweight. Negative results were found in another two lots, the lot with Organic Phosphorus application associated with Vitamin B12 (FOB12) posted a loss of R\$ 1.77 per kg bodyweight, and lot of animals without applying FOB12, $\mathrm{R} \$ 0.75$ per $\mathrm{kg}$ bodyweight. These results have made the termination system feedlot lambs, tested in this trial, uneconomical.
\end{abstract}

Keywords: costs, profitability, lamb finishing. 
Rev. Elet. em Gestão, Educação e Tecnologia Ambiental (e-ISSN: 2236-1170)

\section{INTRODUÇÃO}

A região da campanha era considerada uma potência na produção de ovinos até a década de 1970. Na década de 1980 e 1990 ocorreu uma crise no setor da ovinocultura devido a uma baixa valorização dos produtos obtidos dos ovinos, fator que refletiu em uma queda bruta na população ovina.

Nos últimos anos, entretanto, há um incremento no consumo de carne ovina. A alta na procura por carne de cordeiro exige a utilização de métodos mais rápidos de terminação, como é o caso do confinamento, que reduz o tempo de terminação quando comparado a animais criados de forma extensiva, favorecendo assim toda cadeia produtiva ao longo do ano na medida em que promove a oferta regular de animais, evitando as entressafras.

Segundo Garcia (2004) o consumo per capita de carne/ano no Brasil é de 0,7 quilogramas. A elevação do consumo de carne ovina per capita é notória em relação à década de 1990 , quando estava em 0,5 $\mathrm{Kg}$ por habitante/ano (BEZERRA, 2004), mantendo setor aquecido em constante crescimento e com uma tendência altamente positiva a longo prazo, permitindo, com isso a consolidação e o desenvolvimento da ovinocultura comercial em todo o país.

A produção de carne ovina, conforme Siqueira (1993) tem aumentado recentemente, impulsionada por um mercado consumidor potencialmente grande nos centros urbanos brasileiros. A ovinocultura expande-se agora por vários estados, sobretudo Rio Grande do Sul, Santa Catarina, Paraná, Mato Grosso do Sul, São Paulo e recentemente Minas Gerais.

A produção de carne apresenta-se como uma atividade alternativa capaz de adicionar renda aos negócios, não só dos ovinocultores em si, mas à atividade rural como um todo, independente de ter ou não tradição na criação de ovinos (SILVA, 1999). O acabamento de cordeiros em regime de confinamento não constitui uma prática usual entre os ovinocultores brasileiros, que tradicionalmente adotam o sistema extensivo de produção. Porém, em função das boas perspectivas para a comercialização da carne ovina é necessária a intensificação no processo de terminação de cordeiros, para agilização dos negócios e produção de carne ovina de qualidade. Sendo assim, o confinamento apresenta-se como uma boa alternativa entre os diferentes métodos de alimentação, buscando acrescentar um alto nível de rendimento das carcaças.

É importante salientar que o confinamento deve ser utilizado como uma estratégia, quando há baixa disponibilidade de pastagens de qualidade para os cordeiros ou em períodos de entressafra, onde a demanda por cordeiro é alta e a oferta está baixa. Também, em regiões onde as condições ambientais propiciam uma elevada contaminação dos pastos por parasitas, juntamente com elevadas taxas de lotação, o desmame precoce e a recria e engorda de cordeiros em confinamento, são técnicas de manejo que controlam as perdas causadas pelos vermes, sem necessitar do uso abusivo de vermífugos.

Para que a terminação de cordeiros em confinamento seja economicamente viável, alguns pontos devem ser observados. Entre eles, destacam-se: a duração do confinamento, a utilização de subprodutos, a compatibilização do nível nutricional e do potencial genético do 
Rev. Elet. em Gestão, Educação e Tecnologia Ambiental (e-ISSN: 2236-1170)

animal. Portanto, na implementação desta prática é importante conciliar estes fatores, com vistas ao seu sucesso econômico.

Antes de iniciar uma criação deve haver um levantamento dos ingredientes disponíveis na sua região, para possibilitar o barateamento dos subprodutos utilizados, além de realizar todas as anotações com os gastos envolvidos no seu rebanho, sejam eles diretos ou indiretos.

Um aspecto importante para a gestão rural é um bom acompanhamento de custos de produção. Porém, ainda têm-se dificuldades na organização da propriedade diante aos custos de produção. O controle e a gestão de custos é fundamental, pois facilita aos produtores a tomada de decisão mais lucrativa.

A expressão "custo de produção" contém vários significados. Do ponto de vista do homem de negócios, os custos a serem considerados dependerão da finalidade que se tem em vista e das decisões que se procura tomar. Para fins de análise econômica, o termo "custo" significa a compensação que os donos dos fatores de produção, utilizados por uma firma para produzir determinado produto, devem receber para que eles continuem fornecendo esses fatores à mesma (HOFFMANN et al., 1978).

A identificação dos custos dentro de um processo produtivo só é possível quando implantamos um sistema de contabilidade, este processo está relacionado com o fornecimento de dados de custos para a identificação dos lucros subseqüentes, bem como, um sistema analítico de toda propriedade, desde terras, materiais utilizados, custos fixos e variáveis, custos com mão de obra, entre outros.

A contabilidade de custos, objetiva suprir a administração de uma organização com dados que representem o montante de recursos utilizados para executar as várias fases de seu processo administrativo. Seu papel adquire maior importância quando a organização é inserida dentro do contexto complexo e dinâmico do mercado atual, que exige maior competitividade de seus produtos e serviços (CALLADO \& CALLADO, 1999).

A composição dos custos de produção está descrita na literatura de várias formas, apresentando processos e terminologias diferentes. Os resultados finais passam por diversas análises, identificando desde as despesas diretas até o custo total.

O ovinocultor para realizar sua tomada de decisão, deve ter como base os conceitos de custo de produção e ficar atento aos itens envolvidos com a alimentação, que é a chave do sucesso para que sua atividade seja produtiva e lucrativa; inclusive o mesmo deve checar se o preço de comercialização está compatível no mercado, possibilitando realizar a análise consistente de sua criação.

Estudos conduzidos por Barros (2008), com utilização de suplementação concentrada apresentaram valores de custos relativamente superiores e apontaram prejuízos no confinamento de ovinos.

É muito pertinente a realização de outros estudos de aferição dos custos e da viabilidade econômica da terminação de cordeiros em outras regiões, bem como cálculos periódicos à medida que os preços relativos alteram-se e a eficiência técnica dos insumos evolui.

O objetivo geral do trabalho foi mensurar os custos de produção e a lucratividade de um o confinamento de cordeiros na Campanha Gaúcha a fim de verificar a sua viabilidade, realizando análise econômica geral do sistema de produção. De maneira específica, foram investigados os seguintes pontos: os custos de produção, avaliando os custos fixos, variáveis e totais de cada 
Rev. Elet. em Gestão, Educação e Tecnologia Ambiental (e-ISSN: 2236-1170)

tratamento; o preço médio de mercado para os cordeiros de cada tratamento após a avaliação das carcaças; a lucratividade do sistema de cada um dos tratamentos utilizados na terminação de cordeiros.

\section{METODOLOGIA}

O trabalho teve inicio em março de 2011, em uma propriedade particular de um membro da Associação de Criadores de Ovinos de Dom Pedrito (ACODOPE). Foram confinados 50 cordeiros da raça Corriedale e 10 cordeiros da raça Texel, todos machos castrados com dentição dente de leite e nascidos entre julho a setembro de 2010, com peso variando entre 16 e 30 quilos.

Ao ingressarem no confinamento, os animais foram identificados e separados para distribuição dos tratamentos, pesados e avaliados quanto ao Escore de Condição Corporal (ECC) variando em uma escala de um a cinco, conforme metodologia descrita por Russel et al. (1969). Os animais passaram por um período pré-experimental de 13 dias, para adaptação às instalações, à alimentação e ao manejo diário.

A área utilizada para o confinamento foi de 96 metros quadrados $\left(\mathrm{m}^{2}\right)$, sendo constituída de um galpão de madeira e telhado de zinco e chão batido, dividido em três piquetes, cada piquete abrigando um lote de animais. Para controlar o manejo sanitário e a distribuição da ração, os animais foram divididos em três lotes, denominados lotes A, B e C, de acordo com seu peso médio. Cada lote respeitava uma área de $0,80 \mathrm{~m}^{2} /$ animal para atender o conforto e bem estar dos animais.

Para avaliação econômica, houve uma segunda divisão dos animais a fim de controlar os custos conforme a raça e a aplicação de Vitamina B12 (FOB $\left.{ }_{12}\right)$. Formaram-se os grupos (1) Texel, (2) Corriedale com aplicação de $\mathrm{FOB}_{12}$ e (3) Corriedale sem aplicação de $\mathrm{FOB}_{12}$.

O lote A era composto por 30 animais Corriedale; em 15 deles foi aplicado por via intramuscular profunda o Fósforo Orgânico associado à Vitamina B12 ( $\mathrm{FOB}_{12}$ ), na dosagem recomendada pelo fabricante.

Os animais foram pesados em intervalos de sete dias. Para a pesagem eram submetidos a um jejum de 12 horas a fim de evitar alteração de peso devido ao enchimento ruminal.

O sistema de alimentação foi baseado em uma ração comercial Piratini ${ }^{\circledR}$ da Cooperativa Coopatrigo, destinada para cordeiros em confinamento, e com núcleo da Tortuga. A alimentação foi dividida em duas ofertas diárias, manhã e tarde, sendo fornecido aos cordeiros um percentual de peso vivo crescente de ração. Ao iniciar o período de adaptação os animais receberam feno à vontade e ração correspondendo a $1 \%$ da média de peso do lote. Com o passar dos dias, aumentou-se a quantidade de ração ofertada e reduziu-se a quantidade de volumoso. A cada três dias de adaptação aumentava-se $1 \%$ de ração sobre a média de peso vivo, até atingir $4 \%$ do peso médio do lote.

No decorrer do trabalho houve a necessidade da colocação de cama de maravalha no galpão, no intuito de amenizar o odor de amônia e a compactação do solo pelo pisoteio dos animais. Uma vez por semana era retirada toda maravalha suja e efetuada uma raspagem do solo e desinfecção com utilização de cal virgem para colocação de nova camada de cama. 


\section{REGEXfHFM}

Rev. Elet. em Gestão, Educação e Tecnologia Ambiental (e-ISSN: 2236-1170)

Para a avaliação dos custos do confinamento, escolheu-se uma metodologia adequada ao sistema de produção, ou seja, buscou-se uma composição de custos amparada na literatura de economia e administração rural e que possa ser aplicada na realidade dos produtores rurais, favorecendo assim a identificação da real situação econômica do sistema produtivo.

A estrutura de custos utilizada apresenta os custos fixos definidos como os recursos aplicados que não se incorporam totalmente aos produtos de curto prazo, participando de diversos ciclos produtivos posteriores. O custo fixo é composto por despesas que o produtor incorre mesmo que não esteja produzindo. Enquadram-se nos custos fixos elementos de despesas do produtor, depreciação de máquinas e benfeitorias bem como suas manutenções. 0 custo variável é composto pelas despesas proporcionais à produção. O custo variável é exposto pelas despesas proporcionais à produção, tais como, ração, mão-de-obra, energia, entre outros.

Os custos variáveis desempenham papel crucial na definição do limite inferior do intervalo dentro do qual o preço mínimo deve variar, constituindo-se, no curto prazo, numa condição necessária para que o produtor continue na atividade (CONAB, 2007).

A pesagem realizada na entrada dos animais ao confinamento serviu para efeito do controle de custo da produção relacionado à quantidade de ração fornecida aos animais, possibilitando através disso estimar quantos quilos de ração foram necessários para cada animal atingir o peso de abate. Além disso, foi possível calcular o custo da alimentação como proporção dos custos totais e frente à receita da venda dos cordeiros, e obter o resultado final de lucratividade ou de prejuízo do confinamento.

\section{O cálculo de custos}

Para obtermos os resultados exatos da produção, foram avaliados e representados todos os valores de implantação e funcionamento do confinamento. Os Custos Totais foram divididos como Custos Fixos e Custos Variáveis. Esquematicamente, a classificação de custos empregada é a apresentada no Quadro 1.

\begin{tabular}{|l|l|}
\hline Custos fixos & Custos variáveis \\
\hline Depreciação das instalações & Energia \\
Depreciação dos equipamentos & Ração \\
Juros sobre o capital médio em instalações e & Mão de obra \\
equipamentos & Medicamentos veterinários e vacinas \\
& Despesas de manutenção \\
& Eventuais \\
\hline
\end{tabular}




\section{REGEXfTFSM}

PICCOLI et all, v(11), no 11, p. 2493-2505, JUN, 2013.

Rev. Elet. em Gestão, Educação e Tecnologia Ambiental (e-ISSN: 2236-1170)

\begin{tabular}{|l|l|}
\hline & FUNRURAL \\
Juros sobre o capital de giro \\
Custo de aquisição dos animais
\end{tabular}

Quadro 1. Estrutura de custos empregada

Calculou-se a partir dos dados o custo total médio por quilograma de cordeiro vivo levado ao abate. O custo total médio decorre da soma do custo fixo médio e do custo variável médio, computados de acordo com o quadro acima. O custo total médio é diretamente confrontado com o preço médio recebido pelo cordeiro vivo, permitindo estabelecer o lucro ou prejuízo por quilograma de cordeiro vivo do empreendimento.

Lucro ou prejuízo = preço médio por $\mathrm{Kg}$ - custo total médio por $\mathrm{Kg}$

Para alcançar o custo médio por quilograma de cordeiro vivo, primeiramente obtém-se o custo médio por quilograma de ganho de peso no confinamento, ou seja, a repercussão dos itens do Quadro 1 para cada quilograma de peso ganho por lote de controle, com a exceção dos custos de aquisição dos animais.

Ao custo médio por quilograma de ganho de peso, adiciona-se o gasto com a aquisição dos cordeiros para o confinamento. Afere-se, então o custo médio por quilograma de cordeiro vivo para abate.

Os custos fixos permaneceram os mesmos para todo o rebanho, enquanto os custos variáveis modificaram de acordo com a quantidade de animais de cada grupo de avaliação econômica, tipo de tratamento aplicado ao grupo e o tempo de permanência no confinamento.

\section{RESULTADOS E DISCUSSÃO}

\section{Lote inteiro}

Apresenta-se a seguir o custo médio por quilograma de ganho de peso dos animais em confinamento.

Tabela 1. Custo por quilograma de ganho de peso do Lote Inteiro

\begin{tabular}{lllll}
\hline Item & $\begin{array}{l}\text { Custos/Kg } \\
\text { ganho peso }\end{array}$ & $\begin{array}{l}\text { \% de C } \\
\text { A) Custos fixos }\end{array}$ & $\begin{array}{l}\text { Ganho de peso Ganho de peso } \\
\text { lote }\end{array}$ & $\begin{array}{l}\text { por animal } \\
\text { pana }\end{array}$ \\
1. Depreciação (d) & $\mathbf{0 , 6 9}$ & $\mathbf{0 , 0 5}$ & $\mathbf{4 4 3 , 4}$ & $\mathbf{7 , 8}$ \\
\hline
\end{tabular}


Rev. Elet. em Gestão, Educação e Tecnologia Ambiental (e-ISSN: 2236-1170)

\begin{tabular}{lll}
\hline \multicolumn{1}{c}{ 1.1 d Galpão } & 0,09 & 0,01 \\
1.2 d Bretes & 0,12 & 0,01 \\
1.3 d Balança & 0,23 & 0,02 \\
2. Juros sobre o capital médio & 0,25 & 0,02 \\
B) Custos variáveis & $\mathbf{1 3 , 1 1}$ & $\mathbf{0 , 9 5}$ \\
1. Energia & 0,15 & 0,01 \\
2. Medicamentos & 0,93 & 0,07 \\
3. Mão de obra & 1,82 & 0,13 \\
4. Alimentação & 8,82 & 0,64 \\
5. Manutenção & 0,12 & 0,01 \\
6. Eventuais & 0,59 & 0,04 \\
7. Juros sobre o capital de giro & 0,25 & 0,02 \\
8. FUNRURAL & 0,42 & 0,03 \\
C) Custo total/ Kg ganho peso & $\mathbf{1 3 , 8 0}$ & $\mathbf{1 , 0 0}$ \\
\hline
\end{tabular}

Percebe-se que os custos variáveis são muito significativos, alcançando $95 \%$ do custo total. Destacam-se dentre eles a mão de obra que alcançou $13 \%$ e a alimentação dos animais que representa $64 \%$ do custo total médio.

O custo de ganho de peso total é apresentado abaixo (item $C$ da Tabela 2) e corresponde ao Custo total por quilograma de ganho de peso de $\mathrm{R} \$ 13,80$ (Tabela 1 ) multiplicado pelo ganho de peso do lote que foi de $443,4 \mathrm{Kg}$ (Tabela 2).

Tabela 2. Custo e prejuízo por quilograma de cordeiro vivo do Lote Inteiro

Item Valor (R\$)
A) Receita
8134,78
A.1) Preço médio Kg vivo
4,19
B) Custo de aquisição
4628,7
B.1) Preço médio Kg de aquisição $\quad 3,00$ 
Rev. Elet. em Gestão, Educação e Tecnologia Ambiental (e-ISSN: 2236-1170)
C) Custo ganho de peso total
6117,81
C.1) Custo médio Kg vivo
5,54
D) Prejuízo por Kg vivo
$-1,35$

\section{Grupo 1: Animais da raça Texel}

As Tabelas 3 e 4 apresentam os custos para o Texel. A composição relativa (\%) de custos é muito semelhante ao ocorrido no lote inteiro. Alimentação e Mão de obra continuam como os itens mais significativos.

Tabela 3. Custo por quilograma de ganho de peso do Grupo 1 (Texel)

\section{Item}

A) Custos fixos

1. Depreciação (d)

$1.1 \mathrm{~d}$ Galpão

$1.2 \mathrm{~d}$ Bretes

$1.3 \mathrm{~d}$ balança

2. Juros sobre o capital médio

B) Custos variáveis/ $\mathrm{Kg} \mathrm{GP}$

1. Energia

2. Medicamentos

3. Mão de obra

4. Alimentação

5. Manutenção

6. Eventuais

7. Juros sobre o capital de giro

8. FUNRURAL

$\begin{array}{ll}\text { Custos/ } & \mathrm{Kg} \\ \text { ganho peso de C }\end{array}$

0,69

0,05

0,03

0,44

0,09

0,12

0,23

0,25

14,22

0,01

0,01

0,02

0,02

0,95

0,15

0,01

0,93

0,06

1,82

0,12

9,67

0,65

0,12

0,01

0,63

0,04

0,25

0,02

0,64
Ganho de Ganho de peso peso lote por animal

$46,1 \quad 4,61$ 
Rev. Elet. em Gestão, Educação e Tecnologia Ambiental (e-ISSN: 2236-1170)

\section{C) Custo total/ Kg ganho peso $14,91 \quad 1,00$}

Embora o custo da alimentação por quilograma de ganho de peso tenha sido maior para o Texel comparativamente ao lote inteiro, os animais deste lote foram abatidos mais precocemente (aos 56 dias), o que implicou uma economia no consumo total de ração e reduziu o custo médio de por quilograma vivo. Isto indica que o tempo de confinamento é uma variável economicamente relevante uma vez que o custo do manejo do animal confinado é elevado.

Tabela 4. Custo e prejuízo por quilograma de cordeiro vivo do Grupo 1 (TEXEL)

\begin{tabular}{|c|c|}
\hline Item & Valor (R\$) \\
\hline A) Receita & 1274,40 \\
\hline A.1)Preço médio Kg vivo & 4,05 \\
\hline B) Custo de aquisição & 805,50 \\
\hline B.1) Preço médio aquisição & 3,00 \\
\hline C) Custo ganho de peso total & 687,15 \\
\hline C.1) Custo médio Kg vivo & 4,74 \\
\hline D) Prejuízo por Kg vivo & $-0,69$ \\
\hline
\end{tabular}

\section{Grupo 2: Corriedale com aplicação de $\mathrm{FOB}_{12}$}

Para os animais Corriedale que receberam $\mathrm{FOB}_{12}$ o custo total por quilograma ganho no confinamento foi ligeiramente inferior. Os custos variáveis ficaram um pouco menores comparativamente ao Texel. Entretanto, o desempenho econômico foi pior porque o tempo de permanência no confinamento aumentou. Mais da metade dos animais (oito unidades) foram abatidos com 66 dias de confinamento. O maior tempo e o maior ganho de peso resultaram em prejuízo porque o custo de ganho de peso no confinamento cresce mais rapidamente do que o acréscimo no preço final por quilograma de cordeiro vivo.

Tabela 5. Custo por quilograma de ganho de peso do Grupo 2 (Corriedale com FOB 12 )

\begin{tabular}{llll}
\hline Custos/ & $\mathrm{Kg} \%$ de C & $\begin{array}{l}\text { Ganho de Ganho de peso } \\
\text { pesolote }\end{array}$ \\
ganho peso & & $\begin{array}{l}\text { por animal } \\
\text { Item }\end{array}$
\end{tabular}


REGESAUFSM

PICCOLI et all, v(11), no 11, p. 2493-2505, JUN, 2013.

Rev. Elet. em Gestão, Educação e Tecnologia Ambiental (e-ISSN: 2236-1170)

\begin{tabular}{|c|c|c|c|}
\hline A) Custos fixos & 0,69 & 0,05 & $111,1 \quad 7,41$ \\
\hline 1. Depreciação (d) & 0,44 & 0,03 & \\
\hline $1.1 \mathrm{~d}$ Galpão & 0,09 & 0,01 & \\
\hline $1.2 \mathrm{~d}$ tecnoforte & 0,12 & 0,01 & \\
\hline $1.3 \mathrm{~d}$ balança & 0,23 & 0,02 & \\
\hline 2. Juros sobre o capital médio & 0,25 & 0,02 & \\
\hline B) Custos variáveis/ Kg GP & 14,09 & 0,95 & \\
\hline 1. Energia & 0,15 & 0,01 & \\
\hline 2. Medicamentos & 1,51 & 0,10 & \\
\hline 3. Mão-de-obra & 1,82 & 0,12 & \\
\hline 4. Alimentação & 9,20 & 0,62 & \\
\hline 5. Manutenção & 0,12 & 0,01 & \\
\hline 6. Eventuais & 0,64 & 0,04 & \\
\hline
\end{tabular}

7. Juros sobre o capital de giro $0,25 \quad 0,02$

8. FUNRURAL $0,41 \quad 0,03$

C) Custo total/ Kg ganho peso $14,78 \quad 1,00$

Tabela 6. Custo e prejuízo por quilograma de cordeiro vivo do Grupo 2 (Corriedale com FOB $\mathrm{F}_{12}$ )

Item

A) Receita

A.1) Preço médio Kg vivo

B) Custo de aquisição

B.1) Preço médio aquisição

C) Custo ganho de peso total

C.1) Custo médio Kg vivo

\section{Valor (R\$)}

1968,82

4,10

1178,70

3,00

1642,16

5,87 
Rev. Elet. em Gestão, Educação e Tecnologia Ambiental (e-ISSN: 2236-1170)

D) Prejuízo por Kg vivo $-1,77$

\section{Grupo 3: Corriedale sem aplicação de $\mathrm{FOB}_{12}$}

Neste grupo, um percentual de $70 \%$ dos animais forma abatidos aos 66 dias de confinamento. Comparativamente ao Grupo 2, o resultado foi melhor, uma vez que o custo variável foi menor nos itens medicamentos (não houve despesa com o $F O_{12}$ ) e alimentação, o preço médio recebido foi um pouco maior e o ganho médio de peso foi melhor.

Tabela 7. Custo e prejuízo por quilograma de cordeiro vivo do Grupo 3 (Corriedale sem $\mathrm{FOB}_{12}$ )

\begin{tabular}{|c|c|c|c|c|}
\hline Item & $\begin{array}{l}\text { Custos/Kg de } \\
\text { ganho peso }\end{array}$ & $\% \mathrm{C}$ & $\begin{array}{l}\text { Ganho de } \\
\text { peso lote }\end{array}$ & $\begin{array}{l}\text { Ganho de peso } \\
\text { por animal }\end{array}$ \\
\hline A) Custos fixos & 0,69 & 0,06 & 286,2 & 8,2 \\
\hline 1. Depreciação (d) & 0,44 & 0,04 & & \\
\hline 1.1 d Galpão & 0,09 & 0,01 & & \\
\hline $1.2 \mathrm{~d}$ tecnoforte & 0,12 & 0,01 & & \\
\hline $1.3 \mathrm{~d}$ balança & 0,23 & 0,02 & & \\
\hline 2. Juros sobre o capital médio & 0,25 & 0,02 & & \\
\hline B) Custos variáveis/ Kg GP & 10,14 & 0,94 & & \\
\hline 1. Energia & 0,15 & 0,01 & & \\
\hline 2. Medicamentos & 0,93 & 0,09 & & \\
\hline 3. Mão de obra & 1,82 & 0,17 & & \\
\hline 4. Alimentação & 6,03 & 0,56 & & \\
\hline 5. Manutenção & 0,12 & 0,01 & & \\
\hline 6. Eventuais & 0,45 & 0,04 & & \\
\hline 7. Juros sobre o capital de giro & 0,25 & 0,02 & & \\
\hline 8. FUNRURAL & 0,39 & 0,04 & & \\
\hline C) Custo total/ Kg ganho peso & 10,83 & 1,00 & & \\
\hline
\end{tabular}




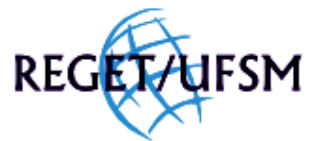

PICCOLI et all, v(11), no 11, p. 2493-2505, JUN, 2013.

Rev. Elet. em Gestão, Educação e Tecnologia Ambiental (e-ISSN: 2236-1170)

O item Alimentação foi muito menor, a ponto dos custos variáveis ficarem relativamente menores (94\%) para este grupo. O custo total por quilograma de ganho de peso foi o menor dos três grupos. Em relação ao Grupo 1, entretanto, o prejuízo foi maior devido ao maior tempo médio de permanência dos animais em confinamento.

Tabela 8. Custo e prejuízo por quilograma de cordeiro vivo do Grupo 3 (Corriedale sem $\mathrm{FOB}_{12}$ )

\begin{tabular}{ll}
\hline Item & Valor (R\$) \\
\hline A) Receita & 4891,10 \\
\hline \multicolumn{1}{c}{ A.1) Preço médio Kg vivo } & 4,27 \\
\hline B) Custo de aquisição & 2643,60 \\
\hline \multicolumn{1}{c}{ B.1) Preço médio aquisição } & 3,00 \\
\hline C) Custo ganho de peso total & 3099,56 \\
\hline \multicolumn{2}{c}{ C.1) Custo médio Kg vivo } \\
\hline E) Prejuízo por Kg vivo
\end{tabular}

O custo de engorda dos animais em confinamento é bastante significativo. Um quilograma de peso ganho no confinamento representa um custo monetário cerca de três vezes superior à receita obtida por um quilograma de cordeiro vivo. O confinamento de cordeiros tem alguma perspectiva de viabilidade econômica se encarado como um acabamento do animal, partindo de um estado nutricional bom para rapidamente deixá-lo em condições de abate, justamente no período de costumeira entressafra. O tempo de manutenção do animal no confinamento precisa ser abreviado, o que implica o uso de animais de boa carga genética para a conversão alimentar, um bom estado nutricional no início do confinamento e constituição de lotes homogêneos em suas características genéticas e de condição corporal. Outros dois fatores que contribuirão para o encaminhamento de um resultado positivo serão a diluição da mão de obra por maior número de animais manejados e o barateamento relativo da alimentação. $O$ último item, a redução do custo da ração, poderá vir de um aumento da eficiência alimentar bem como da redução do preço do quilograma da ração. A experiência histórica de outras cadeias produtivas nas quais as empresas insumidoras foram muito importantes no desenvolvimento tecnológico redutor de custos, tais como suínos e frangos, sugere uma promissora capacidade de soluções a ser oferecida aos criadores. 
Rev. Elet. em Gestão, Educação e Tecnologia Ambiental (e-ISSN: 2236-1170)

\section{CONCLUSÕES}

O estudo indicou prejuízo no confinamento de cordeiros na Campanha Gaúcha nas condições técnicas enfrentadas e de mercado do período. Tal circunstância obriga a uma reflexão, com identificação dos itens mais importantes na composição dos custos e alterações de manejo para alcançar uma rentabilidade positiva.

Os custos variáveis são muito significativos na composição do custo total, especialmente os itens alimentação e mão de obra. Alterações técnicas que minimizem a quantidade de uso destes fatores de produção ou a substituição por elementos de alimentação com preços mais baixos terão grande repercussão na redução dos custos totais de produção.

O Grupo 1, composto de animais Texel, embora tenha incorrido em prejuízo como os demais grupos, apresentou o melhor desempenho econômico. O reduzido tempo de permanência no confinamento foi um fator importantíssimo para o melhor rendimento relativo.

Nas condições do estudo, o Fósforo Orgânico associado à Vitamina B12 não se mostrou viável economicamente.

\section{REFERÊNCIAS BIBLIOGRÁFICAS}

BARROS, C. S. Análise econômica de sistemas de produção de ovinos para carne. Curitiba: UFPR, 2008. 144p. Dissertação (Mestrado em Ciências Veterinárias), Universidade Federal do Paraná, 2008.

BEZERRA, J. A. Revolução Sertaneja. Revista Globo Rural, São Paulo, n. 228, ano 20, p. 20-26, 2004.

CALLADO, A. A. C.; CALLADO. A. L.C. Custos: um desafio para a gestão do agronegócio. In: CONGRESSO BRASILEIRO DE CUSTOS, 6., 1999, São Paulo. Anais... São Paulo: FEA UUSP, 1999.

CONAB. Metodologia de cálculo de custos de produção, 2007. Disponível em:<http://www.conab.gov.br/conabweb/download/safra/custosdeproduçãometodologia.pdf>. Acesso em: 26 jan. 2011.

GARCIA, C. A. Ovinocultura e Caprinocultura. Marília: Universidade de Marília, 2004. 22 f. Apostila.

HOFFMANN, R. et al. Administração da empresa agrícola. São Paulo: Pioneira, 1978. 325 p.

RUSSEL, A. J. F.; DONEY, J.M.; GUNN,R.G. Subjective assessment of body fat in live sheep. Journal of Agriculture Science, Savoy, v.72, p.451-454, 1969.

SIQUEIRA, E. R. Confinamento:a receita dos paulistas para engordar cordeiros. A Granja, v. 49, p. 12 -17, 1993

SILVA, L. F. Crescimento, composição corporal e exigências nutricionais de cordeiros abatidos com diferentes pesos. Santa Maria: UFSM, 1999. 70 p. Dissertação (Mestrado em Zootecnia), Universidade Federal de Santa Maria, 1999 